\title{
Impact of Phyllanthus amarus extract on antioxidant enzymes in Drosophila melanogaster
}

\author{
N. Manasa, J. S. Ashadevi* \\ Department Of Zoology, Yuvaraja's College (Autonomous), University Of Mysore, Mysore, Karnataka, India.
}

\begin{tabular}{|c|c|}
\hline ARTICLE INFO & ABSTRACT \\
\hline $\begin{array}{l}\text { Article history: } \\
\text { Received on: } 15 / 10 / 2015 \\
\text { Revised on: } 28 / 10 / 2015 \\
\text { Accepted on: } 18 / 11 / 2015 \\
\text { Available online: } 19 / 12 / 2015\end{array}$ & $\begin{array}{l}\text { Oxidative stress (OS) leads to deleterious effects in organisms. Main cause for the oxidative stress is the } \\
\text { generation of free radicals in the cells. Antioxidants have ability to scavenge and neutralize the free radicals. } \\
\text { Therefore, present study has undertaken to test the antioxidant potential of Phyllanthus amarus ethanolic extract } \\
\text { (PAE) in Drosophila melanogaster through biochemical analysis. OS test has been conducted in the PAE } \\
\text { supplemented different age grouped flies by exposing to } 20 \mathrm{mM} \text { acrylamide (ACR). Antioxidant enzymes such as } \\
\text { superoxide dismutase (SOD), catalase (CAT) and reduced glutathione (GSH) were measured in different age }\end{array}$ \\
\hline $\begin{array}{l}\text { Key words: } \\
\text { Phyllanthus amarus, } \\
\text { Drosophila melanogaster, } \\
\text { Oxidative stress, Acrylamide, } \\
\text { antioxidant enzymes }\end{array}$ & $\begin{array}{l}\text { grouped stressed and non-stressed PAE supplemented flies. OS test results revealed that PAE II treated groups } \\
\text { showed significantly increased ACR resistance when compared to PAE I and control groups. All the age grouped } \\
\text { PAE supplemented flies in non-stress conditions had significantly high SOD and CAT activities. Highest SOD } \\
\text { and CAT activities were observed in PAE II treated } 30 \text { and } 45 \text { days flies respectively. Under stress conditions, } \\
\text { the elevated levels of SOD activities were found in } 30 \text { and } 45 \text { days and CAT activities in } 30,45 \text { and } 60 \text { days aged } \\
\text { PAE treated flies. Overall data highlights that there is a dose dependant relationship between the extract } \\
\text { concentrations and enzyme activities. }\end{array}$ \\
\hline
\end{tabular}

\section{INTRODUCTION}

Antioxidants are micronutrients that have gained importance due to their ability to scavenge and neutralize the free radicals before causing damage to body cells. Many naturally occurring antioxidant compounds have been identified as free radical scavengers [1]. Antioxidant enzymes play an important role in lowering the reactive oxygen species (ROS) levels and reduces the oxidative stress. The antioxidant enzymes such as superoxide dismutase (SOD), catalase (CAT), ascorbate peroxidase and glutathione reductase $(\mathrm{GSH})$, acts as natural defense mechanism against oxidative stress in biological systems [2]. Medicinal plants are considered to be the best source for antioxidant compounds [3]. As it produces the significant amount of antioxidants to prevent the oxidative stress caused by

\footnotetext{
* Corresponding Author

Ashadevi J.S., Assistant Professor, Department of Zoology, Yuvaraja's College (Autonomous), University of Mysore, Mysore-570005, India

Contact:09448258374. Email: jsaycm15@gmail.com
}

photons and oxygen, they are considered as potential source of new compounds with antioxidant activity [4]. Secondary metabolites of plants such as flavonoids and polyphenols exhibited a significant biological role due to their antioxidants activity [5, 6]. Phenolic and flavonoid-rich antioxidants were abundantly found in both edible and non-edible plants. Phyllanthus amarus is considered as one such plant species used in Indian Ayurveda enriched with polyphenolic compounds [7]. It is a rejuvenative herb belongs to the family Phyllanthaceae. In vitro antioxidant activity of $P$. amarus has been reported by few studies [7,8]. The hepatoprotective, antidiabetic, antiulcer and anticancer activities of $P$. amarus are also been reported [9]. The ethanolic extract of $P$. amarus leaves exhibited hypoglycemic activity on alloxan-induced diabetic mice [10].

Recently, we have reported that the ethanolic extract of $P$. amarus species extends the lifespan in Drosophila melanogaster [11]. Since the mechanism by which $P$. amarus enhances the lifespan in Drosophila is not yet known. Therefore, in order to understand such mechanism, we performed the few antioxidant enzymatic assays in Phyllanthus amarus ethanolic extract (PAE) supplemented flies under stress and non-stress conditions. 


\section{MATERIALS AND METHODS}

Acrylamide (ACR), Nitro blue tetrazolium (NBT), Hydrogen peroxide, Nicotinamide adenine dinucleotide phosphate (NADP), Trichloroacetic acid (TCA), Reduced glutathione, 5, 5'dithio-bi's (2-nitrobenzoic acid) (DTNB), Bovine serum albumin (BSA) were procured from Himedia, Mumbai, India.

\subsection{Extract preparation}

Phyllanthus amarus was collected from natural habitat in Mysore, Karnataka, India. The extract was prepared by using the aerial parts of the plant. The powder form of the plants was subjected to Soxhlet unit to get ethanolic extraction. The collected dried extracts were stored and used for the analyses. All the experiments were carried out using two different concentrations of the PAE (dose-I: $1 \mathrm{mg} / \mathrm{ml}$ and dose-II: $10 \mathrm{mg} / \mathrm{ml}$ ).

\subsection{Drosophila culture}

Wild stocks of D. melanogaster (Oregon K strain) flies were procured from Drosophila Stock Center, Department of Zoology, University of Mysore, Mysore, Karnataka. The flies were cultured and maintained in standard 'Wheat cream agar media' seeded with dry yeast granules. Further, flies were multiplied by subculture and maintained at $22 \pm 1^{\circ} \mathrm{C}$ with relative humidity of $60-70 \%$.

Synchronized eggs were collected from the Delcour technique as per the standard procedure [12] and newly eclosed flies were segregated under mild anesthesia. Then, flies were maintained in PAE supplemented culture media and aged them for $15,30,45$ and 60 days.

\subsection{Oxidative Stress (OS) Test}

The OS test was carried out by following filter paper disc method [13]. The OS test was conducted by the induction of ACR in the PAE supplemented flies at the age group of 15, 30, 45 and 60 days.

The flies were induced with $20 \mathrm{mM}$ ACR was employed along with 5\% sucrose in a vial of size $9 \times 3 \mathrm{~cm}$ containing 5-6 layers of filter papers in different batches (20 flies /vial). Then mortality rates were recorded until all the flies attain death and the rate of survival was recorded with an interval of 6 hour. Extract supplemented flies were considered as treated groups and only yeast fed flies were considered as control group. Hundred flies were maintained in each group.

\subsection{Biochemical Estimations}

The activity of few antioxidant enzymes namely, SOD, CAT and GSH were measured in stress and non-stress conditions. The extract supplemented with OS induced flies were considered as stress group and the extract supplemented without OS induced flies were considered as non-stress group.

The enzyme activity was studied in different age grouped $(15,30,45$, and 60 days) PAE treated flies in two concentrations. The whole body homogenate was prepared by using $0.1 \mathrm{M}$ sodium-phosphate buffer (pH7.4). Then the samples were centrifuged at 2,000 rpm for 10 minutes and the supernatant was used for measuring SOD and CAT activities. The enzyme activities were measured in triplicates in each group.

\subsubsection{Superoxide dismutase (SOD)}

SOD activity was measured by using NBT method [14]. Three $\mathrm{ml}$ reaction mixture was prepared by adding $50 \mathrm{mM}$ potassium phosphate buffer ( $\mathrm{pH} 7.8$ ), $13 \mathrm{mM}$ methionine, $2 \mu \mathrm{M}$ riboflavin, $0.1 \mathrm{mM}$ EDTA, $75 \mu \mathrm{M}$ NBT and $50 \mu \mathrm{L}$ of enzyme extract.

All the tubes were exposed to $400 \mathrm{~W}$ bulbs for $15 \mathrm{~min}$. and then absorbance was read at $560 \mathrm{~nm}$. The $50 \%$ inhibition of the reaction between riboflavin and NBT in the presence of methionine was considered as one unit of SOD activity and it was expressed in units/mg of protein.

\subsubsection{Catalase (CAT)}

CAT activity was measured by following the standard protocol [15]. The activity was measured based on the quantity of the $\mathrm{H}_{2} \mathrm{O}_{2}$ substrate remaining after the action of CAT present in the enzyme extract. To measure this, $0.4 \mathrm{ml}$ of enzyme extract was mixed with $2.6 \mathrm{ml}$ of phosphate buffer along with $30 \%$ of $\mathrm{H}_{2} \mathrm{O}_{2}$. The activity was measured by determining the decomposition of $\mathrm{H}_{2} \mathrm{O}_{2}$ at $240 \mathrm{~nm}$. CAT activity was calculated by using the millimolar extinction coefficient of 43.6 and expressed in terms of $\mu \mathrm{m} / \mathrm{min} / \mathrm{mg}$ of protein.

\subsubsection{Reduced glutathione (GSH)}

The activity of GSH was measured by using DTNB method [16]. To measure the enzyme activity, flies were homogenized in ice-cold 10\% TCA and 10 mM EDTA solutions $(1: 1)$.

Then, homogenate was centrifuged at $5000 \mathrm{rpm}$. Further, enzyme reaction mixture was prepared by adding $200 \mu \mathrm{l}$ of supernatant, $0.2 \mathrm{M}$ Tris-buffer (pH8.0) and $50 \mu \mathrm{l}$ of DTNB. The reaction mixture was incubated for $10 \mathrm{~min}$. at room temperature to get yellow coloured complex. The absorbance was read at $412 \mathrm{~nm}$ and the activity was expressed as $\mu \mathrm{g} / \mathrm{mg}$ protein.

\subsubsection{Estimation of protein}

Protein estimation was quantified as per the standard procedure [17]. For this, $20 \mu 1$ of the homogenate was mixed with Lowry's reagent and Folin-Ciocolteu's solution. The optical density was measured at $660 \mathrm{~nm}$ against blank after incubation. Then calculated the amount of protein in each group with the BSA standard graph.

\subsection{Statistical Analysis}

All the enzyme activities were statistically analyzed using the SPSS software, version 20.0 and the values were expressed in mean \pm SE. The significance differences among the analyzed groups were compared by one-way ANOVA followed by DMRT. Values of $p<0.05$ considered as statistically significant. 


\section{RESULTS}

\subsection{Effect of PAE on ACR resistance in D. melanogaster flies}

The PAE supplemented flies of age group 15, 30, 45 and 60 days were exposed to ACR for the induction of OS. The result obtained from OS test of different aged flies were represented in figure 1 . The resistance ability was significantly increased in the 15 days age group of both PAE I and PAE II supplemented flies and 45 days of PAE II supplemented flies when compared to control group. However, the pattern of stress induction was same in 30 days and 60 days aged PAE treated and control groups. Further, it reveals that PAE II treated groups showed high ACR resistance ability when compared to PAE I and control groups.

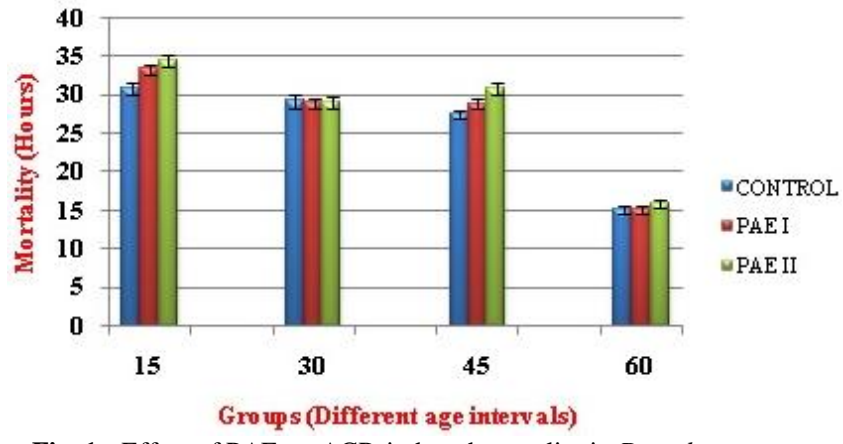

Fig. 1: Effect of PAE on ACR-induced mortality in D. melanogaster.

\subsection{Biochemical Estimations}

\subsubsection{SOD}

The SOD activity was measured in stress and nonstressed group of PAE fed flies are compiled in figure 2. The activity was measured in the flies with different age intervals.

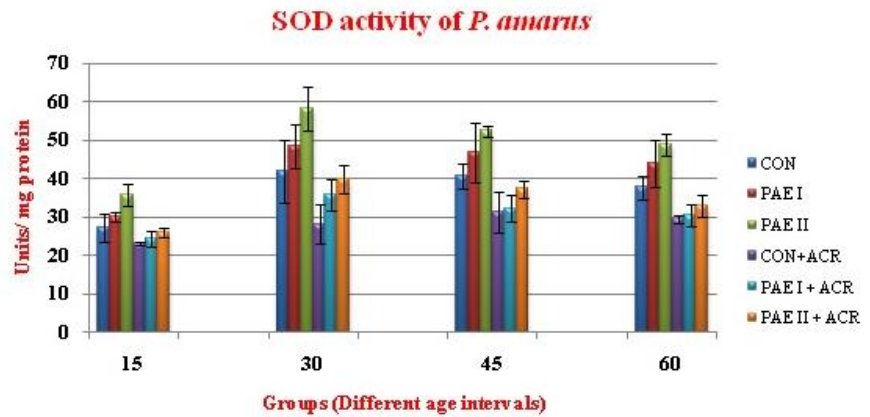

Fig. 2: SOD activity in control and PAE prefed flies before and after exposre to ACR.

The enzyme activity in non-stress state of all the analyzed group was comparatively lower at 15 days aged flies, shows maximum at 30 days, then gradually declined in 45 and 60 days age grouped flies. It was found that all the age groups of PAE treated flies of both the dose treatments had significantly higher enzyme activity than control groups. PAE II has maximum SOD activity in all the age groups. The maximum enzyme activity of PAE II was observed at the age group of 30 days (58.10 units/mg of protein). Under stress condition, ACR induced control flies of all the age group has reduced the SOD activity than PAE treated groups. In both the doses of PAE supplemented stressed fly groups, the enzyme activity was comparatively lower at 15 days and increased to maximum at 30 days. Further, SOD activity was suddenly decreased in PAE I and gradually decreased in PAE II treated stress group at 45 and 60 days. Similar to non-stress groups, PAE II supplemented stress group has maximum enzyme activity at the age of 30 days (39.82 units/mg of protein). Further result reveals that in both stress and non-stress conditions, the PAE II showed maximum SOD activity in all the age groups.

\subsection{2. $C A T$}

The CAT activity was measured in PAE prefed flies of stress and non-stressed groups are represented in figure 3 . The activity was expressed as $\mu \mathrm{m} \mathrm{H}_{2} \mathrm{O}_{2}$ consumed/min/mg of protein. The enzyme activity of PAE treated groups was gradually increased from 15 to 30 days aged flies and showed maximum activity in 45 days, then declined in 60 days aged flies.

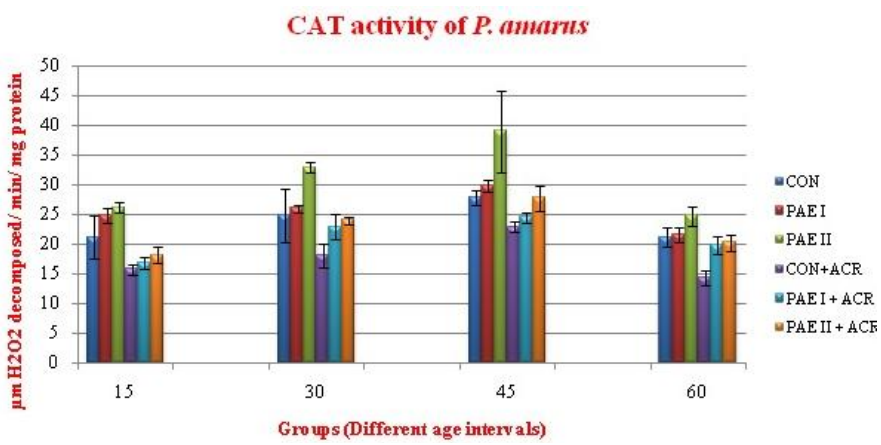

Fig. 3: CAT activity in control and PAE prefed files before and after exposure to ACR.

It was found that the enzyme activity in all the age groups of PAE treated flies was significantly increased compare to control group. Further, it reveals that PAE II treatment groups had highest CAT activity in all the age groups, with the maximum activity at 45 days $\left(38.93 \mu \mathrm{m} \mathrm{H}_{2} \mathrm{O}_{2}\right.$ consumed $/ \mathrm{min} / \mathrm{mg}$ of protein). Similar observation was found in ACR induced stress groups, where PAE treated groups had higher CAT activity compared to ACR with control. In both the doses of PAE treated stress groups, enzyme activity was gradually increased from 15 to 30 days and reached maximum in 45 days. Whereas in both the doses of PAE treated 60 days stress groups, enzyme activity was declined. Similar to non-stressed conditions, the PAE II has maximum CAT activity in all the age groups except in 60 days in stress conditions.

\subsubsection{GSH}

The GSH activity was measured in PAE prefed flies of stress and non-stressed groups are shown in figure 4. The data reveals that GSH activity of the PAE I in non-stressed groups was increased significantly in 15 and 45 days when compared to control group. PAE II supplemented flies have significantly increased GSH activity in all the age group flies except in 60 days, where activity was declined. However, the maximum enzyme activity was observed in PAE II at 45 days (77.77 units/mg of 
protein). On ACR treatment, the GSH activity of PAE treated flies was found to be lower when compared to control except in PAE II treated 60 days flies where the activity was significantly increased.

\section{GSH activity of $P$. amaris}

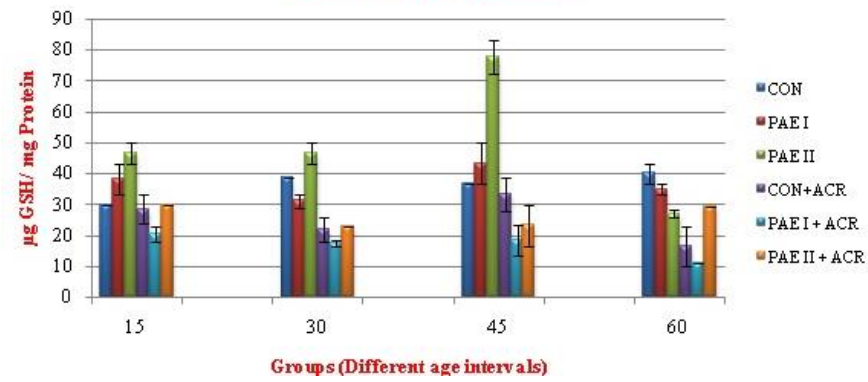

Fig. 4: GSH activity in control and PAE prefed files before and after exposure to ACR.

\section{DISCUSSION}

Oxidative stress leads to deleterious effects on the physiology of organisms. Main cause for the oxidative stress is the generation of reactive oxygen species in the cells and later they are converted into free radicals [18]. It has been reported that the cranberry diet reduces the oxidative stress and extend the lifespan [19]. ACR is commonly used as a toxicant to generate oxidative stress in model organisms [13]. In the present study, OS test was carried out for induction of stress by using ACR. PAE supplemented flies when undergone OS, showed prolonged survival time than control flies. The resistance ability for ACR was increased in PAE II by $11.36 \%$ in 15 days age grouped flies and by $12.66 \%$ in 45 days age grouped flies. The PAE II supplemented flies showed more resistance ability with high degree of survival time than PAE I treatment. Thus, oxidative stress resistance ability of PAE was validated from the current study.

The endogenous antioxidant system can scavenge the excess amount of ROS. The major antioxidant enzymes such as SOD, CAT and GSH are serving as primary endogenous antioxidants to deactivate the ROS [20]. The most common ROS is $\mathrm{O}_{2}$ which is easily dismutated either through non-enzymatically or by enzymatically to $\mathrm{H}_{2} \mathrm{O}_{2}$. The $\mathrm{H}_{2} \mathrm{O}_{2}$ is generated in the cells under normal as well as wide range of stressful conditions. $\mathrm{H}_{2} \mathrm{O}_{2}$ has no unpaired electrons, unlike other oxygen radicals. It can readily cross biological membranes and consequently can cause oxidative damage. Further, $\mathrm{H}_{2} \mathrm{O}_{2}$ is converted to $\mathrm{H}_{2} \mathrm{O}$ by CAT or GSH. As organism get aged or under oxidative stress conditions, the scavenging capacity of the body is enormously decreased and thus could attack the intracellular molecules and ultimately lead to oxidative damage [21, 22].

The antioxidants found in many medicinal plants and herbs are capable of augmenting the antioxidant enzyme activities, their by it scavenges the free radicals. The dietary consumption of Hawthorn extracts showed beneficial impact on endogenous antioxidant enzymes [23]. It has been shown that the antioxidant activity of Emblica officinalis increases SOD, CAT and glutathione peroxidase (GPx) in rat [24]. Tinospora cordifolia is a well-known antioxidant rich medicinal plant used in Indian Ayurveda. The root extract of $T$. cordifolia increases SOD, GSH and CAT activities in diabetic rats [25]. Syzygium cumini an antioxidant rich plant increases GSH, SOD and CAT activity in rat [26]. Further, it has been proved that curcumin of Curcuma longa is capable of scavenging free radicals by increasing SOD, CAT and GPx [27].

In the present study SOD, CAT and GSH activities were measured in different age grouped PAE extract supplemented flies. The activities were measured in stressed and non-stressed state. Enzyme activities were gradually increasing, then decreased as the age increased.

The SOD activity was reached peak at 30 days and the CAT activity reached peak at 45 days in both stress and non-stress conditions of all the analyzed groups. Further, it was observed that PAE treated flies have high SOD and CAT activities in all the age grouped flies. Similar such observation was noticed even in extract fed flies induced with ACR. The data on GSH enzyme study summarizes that only 45 days aged non-stressed PAE II has significant amount of enzyme activity and there was no significant observation was exist in GSH activity of rest of the age group flies. Based on these result it indicates that the antioxidants of PAE acts as free radical scavenging molecules by increasing SOD and CAT.

Increasing the activity of antioxidant enzymes is also expected to increase longevity. It has been shown that the anthocyanins of Xinjiang black mulberry fruit prolongs the lifespan in Drosophila with increased activities of GPx, SOD and CAT [28]. Further, it has been reported that the activities of antioxidant enzymes might be one of the reasons for prolonging the lifetime of D. melanogaster. The supplementation of apple polyphenol increases the lifespan of D. melanogaster by regulating SOD and CAT genes [29]. In our earlier report [11], we have shown that PAE II prolongs the life span of $D$. melanogaster by $75 \%$. In the present investigation, we have demonstrated that the supplementation of PAE is associated with the elevation of SOD and CAT activities. Thus, PAE extends the life span of fruit fly by increasing SOD and CAT activities. Present result is similar to the findings of earlier studies conducted on anthocyanins of black mulberry and apple polyphenols on Drosophila life span extension [28, 29].

In conclusion, the studies reveal that the supplementation of PAE increases the resistance ability against oxidative stress molecule. It increases the SOD and CAT activities even under stress conditions, thus it prolongs the longevity in $D$. melanogaster. Therefore, PAE may present a viable and potentially powerful therapy for aging and age-related diseases.

\section{ACKNOWLEDGEMENTS}

The first author is thankful to Indian Council of Medical Research, New Delhi for the financial assistance received in the form of SRF. 


\section{REFERENCES}

1. Zheng W, Wang SY. Antioxidant activity and phenolic compounds in selected herbs. Journal of Agriculture and Food Chemistry. 2001; 49: 5165-5170.

2. Beyer W, Imlay J, Fridovich I. Superoxide dismutases. Progress in Nucleic Acid Research and molecular biology. 1991; 40: 221-253.

3. Hasani P, Yasa N, Ghanbari SV, Mohammadirad A, Dehghan G, Abdollahi M. In vivo antioxidant potential of Teucrium polium, as compared to a-tocopherol. Acta Pharmaceutica. 2007; 57: 123-129.

4. Ali SS, Kasoju N, Luthra A, Singh A, Sharanabasava H, Sahu A, Bora U. Indian medicinal herbs as sources of antioxidants. Food Research International. 2008; 41: 1-15.

5. Agati G, Matteini P, Goti A, Tattini M. Chloroplast located flavonoids can scavenge singlet oxygen. New Phytologist. 2007; 174: 77-89.

6. Huda-Fanjan N, Noriham A, Norrakiah AS, Babji AS. Antioxidant activity of plants methanolic extracts containing phenolic compounds. African Journal of Biotechnology. 2009; 8(3): 484-489.

7. Kumaran A, Karunakaran RJ. In vitro antioxidant activities of methanol extracts of five Phyllanthus species from India. LWT-Swiss Society of Food Science and Technology. 2007; 40: 344-352.

8. Kumara KKS, Chethan J, Manasa N, Ashadevi JS, Prakash HS. Bioactive potential of herbaceous Phyllanthus species. International Journal of Pharmacy and Pharmaceutical Sciences, 2012; 4(4): $457-$ 461.

9. Thyagarajan SP, Jayaram S, Gopalakrishnan V, Hari R, Jeyakumar P, Sripathi MS. Herbal medicines for liver diseases in India. Journal of Gastroenterology and Hepatology. 2002; 17: S370-S376.

10. Shetti AA, Sanakal RD, Kaliwal BB. Antidiabetic effect of ethanolic leaf extract of Phyllanthus amarus in alloxan-induced diabetic mice. Asian Journal of Plant Science and Research. 2012; 2(1): 11-15.

11. Manasa N, Ashadevi JS. Herbaceous Phyllanthus species extracts promotes longevity in Drosophila melanogaster. International Journal of Research in Phytochemistry and Pharmacology. 2015; 4(1): 1-9.

12. Ramachandra NB, Ranganath HA. Multifaceted approach to evaluate the relationship among closely related forms of Drosophila. Genome. 1988; 30: 58

13. Prasad SN, Muralidhara. Neuroprotective effect of geraniol and curcumin in an acrylamide model of neurotoxicity in Drosophila melanogaster: Relevance to neuropathy. Journal of Insect Physiology. 2014; 60: 7-16.

14. Beauchamp C, Fridovich I. Superoxide dismutase: Improved assays and an assay applicable to acrylamide gels. Analytical Biochemistry. 1971; 44: 276-287.

15. Aebi H. Catalase. In: Bergmeyar HU, editor. Methods in enzymatic analysis. New York: Academic Press; 1984, p. 674-684.

16. Ellman GL. Tissue sulfhydryl groups. Archives of Biochemistry and Biophysics. 1959; 82: 70-77.

17. Lowry OH, Rosebrough NJ, Farr AL, Randall RJ. Protein measurement with folin phenol reagent. Journal of Biological Chemistry. 1951; 193(1): 265-275.

18. Ara N, Nur H. In vitro antioxidant activity of methanol leaves and flowers extracts of Lippia alba. Research Journal of Medicine and Medical Sciences. 2009; 4: 107-110.
19. Sun Y, Yolitz J, Alberico T, Sun X, Zou S. Lifespan extension by cranberry supplementation partially requires SOD2 and is life stage independent. Experimental Gerontology. 2014; 50: 57-63.

20. Wills NK, Kalariya N, Ramanujam VMS, Lewis JR, Abdollahi SH, Husain A, van Kuijk, FJ. Human retinal cadmium accumulation as a factor in the etiology of age-related macular degeneration. Experimental Eye Research. 2009; 89: 79-87.

21. Minotti G. Metals and membrane lipid damage by oxy-radicals. Annals of the New York Academy of Sciences. 1988; 551: 34-44.

22. Lee KS, Lee BS, Semnani S, Avanesian A, Um CY, Jeon HJ, Seong $\mathrm{KM}, \mathrm{Yu} \mathrm{K}$, Min KJ, Jafari M. Curcumin extends life span, improves health span, and modulates the expression of age- (associated aging genes) in Drosophila melanogaster. Rejuvenation Research. 2010; 13: 561-570.

23. Zhang Y, Shen T, Liu S, Zhao J, Chen W, Wang H. Effect of Hawthorn on Drosophila melanogaster Antioxidant-Related Gene Expression. Tropical Journal of Pharmaceutical Research. 2014; 13(3): 353-357.

24. Bhattacharya A, Chatterjee A, Ghosal S, Bhattacharya SK. Antioxidant activity of active tannoid principles of Emblica officinalis (amla). Indian Journal of Experimental Biology. 1999; 37: 676-680.

25. Prince PSM, Menon VP. Antioxidant action of Tinospora cordifolia root extract in alloxan diabetic rats. Phytotherapy Research. 2001; 15(3): 213-8.

26. Prince PSM, Menon VP, Pari L. Hypoglycaemic activity of Syzigium cumini seeds: effect on lipid peroxidation in alloxan diabetic rats. Journal of Ethnopharmacology. 1998; 61(1): 1-7.

27. Reddy ACP, Lokesh BR. Studies on spice principles as antioxidants in the inhibition of lipid peroxidation of rat liver microsomes. Molecular and Cellular Biochemistry. 1992; 111: 117-124.

28. Jiang Y. Effects of anthocyanins derived from Xinjiang black mulberry fruit on delaying aging. Wei Sheng Yan Jiu. 2010; 39(4): 451-3.

29. Peng C, Chan HYE, Huang Y, Yu H, Chen ZY. Apple Polyphenols Extend the Mean Lifespan of Drosophila melanogaster. Journal of Agricultural and Food Chemistry. 2011; 59(5): 2097-2106.

How to cite this article:

Manasa N. and Ashadevi J.S. Impact of Phyllanthus amarus extract on antioxidant enzymes in Drosophila melanogaster. J App Biol Biotech, 2015; 3 (06): 043-047. DOI: 10.7324/JABB.2015.3607 